\title{
A clinicopathologic study of mucinous adenocarcinoma of the stomach
}

\author{
Hideki Kawamura, Yukifumi Kondo, Shohei Osawa, Yasunori Nisida, Kuniaki Okada, Hiroyuki Isizu, \\ Takasi Uebayasi, Manabu Takahasi, and Tunetake Hata \\ Department of Surgery, JA Sapporo Kosei Hospital, Sapporo, Japan
}

\begin{abstract}
Background. Mucinous adenocarcinoma of the stomach is a rare histologic type of gastric adenocarcinoma and its features are still controversial. We attempted to clarify the clinicopathologic characteristics of this histologic type.

Methods. We reviewed the records of 112 patients with mucinous adenocarcinoma of the stomach (MUC) and 4160 patients with nonmucinous gastric adenocarcinoma (NMUC) for factors including age; sex; tumor location, size, and depth; lymph node metastasis; lymphatic or venous permeation; peritoneal dissemination; liver metastasis; and survival rate. We also investigated the relationship between cancer depth and lymph node metastasis. Statistical analysis included $\chi^{2}$ and Student's $t$-tests. Survival rates were calculated by the KaplanMeier method and analyzed by the log rank test.

Results. MUC was found mostly in younger patients, in the lower part of the stomach and was of larger size, invading to or beyond the muscularis propria, positive for lymph node involvement, and associated with peritoneal dissemination. The overall 5-year survival rate in patients with MUC was lower than that in NMUC patients, because the MUC patients more frequently had advanced stage disease; however, the survival rates in the two groups did not differ significantly. As for the relationship between cancer depth and lymph node involvement, the frequency of lymph node metastasis increased when invasion reached the submucosa in patients with MUC compared with those with NMUC while the frequency of lymph node metastasis increased in the muscularis propria in patients with NMUC compared with those with NMUC.

Conclusion. MUC was characteristically found in younger patients, at lower sites, at an advanced stage, and with peritoneal dissemination; MUC had a poor prognosis. Lymph node metastasis should be suspected when MUC invades to the submucosa or deeper.
\end{abstract}

Key words Stomach neoplasms $\cdot$ Adenocarcinoma $\cdot$ Mucin

Offprint requests to: $\mathrm{H}$. Kawamura

Department of Surgery, Tomakomai City General Hospital, 1-2-21, Honkou-cho, Tomakomai, Hokkaido 053-8567, Japan Received: March 14, 2001 / Accepted: June 18, 2001

\section{Introduction}

Adenocarcinomas of various organs sometimes produce a mucinous component, and this is also observed in gastrointestinal tract cancers. Mucinous adenocarcinoma occurs more frequently in the colorectum than in the stomach. Therefore, there are many reports of mucinous adenocarcinoma of the colorectum [1-4]. These reports indicate that the frequency of mucinous adenocarcinoma of the colorectum is $5.7 \%-11.7 \%$ of colorectal cancers, with histopathologic features such as larger tumor size, deep invasion, and poor prognosis. However, mucinous adenocarcinoma of the stomach (MUC) is a very rarely found histologic type $(2.4 \%-$ $4.9 \%$ ) of gastric cancer [5-12]. Therefore, there are few reports on MUC and little is clear about its clinicopathologic characteristics and prognosis.

To clarify the features of MUC, we conducted a retrospective investigation comparing MUC with nonmucinous gastric adenocarcinoma (NMUC).

\section{Patients and methods}

The subjects were 4272 patients who had undergone surgery at the Department of Surgery, JA Sapporo Kosei Hospital, from January 1970 to December 1998. Of these 4272 patients, 112 were classified as having as MUC (i.e., the tumors histologically showed $50 \%$ or more mucin pools). We examined the patients' ages; sex; tumor location, diameter, and depth of invasion; lymph node metastasis; lymphatic or venous permeation; peritoneal dissemination; liver metastasis; and survival rates. To determine the relationship between cancer depth and lymph node metastasis, we examined the frequency of lymph node metastasis in patients showing various degrees of cancer depth. Staging and the histological typing were classified according to the Japanese classification of gastric carcinoma proposed 
by the Japanese Research Society for Gastric Cancer [13].

Statistical analysis was carried out using $\chi^{2}$ and Student's $t$-tests. Survival rates were calculated by the Kaplan-Meier method and analyzed by log rank test. Differences were considered significant when the $P$ value was less than 0.05 .

\section{Results}

\section{Clinicopathologic factors}

Of the 4272 patients, 112 had MUC (2.6\%), the lowest proportion of the common types of gastric cancer (Table 1). MUC patients were younger than NMUC patients, and MUC tumors were in a lower stomach location and had a larger size than NMUC tumors. Histologically, MUC showed deeper invasion to the gastric wall, as well as higher frequencies of lymph node metastasis, lymphatic and venous permeation, and peritoneal dissemination (Table 2). No significant differences

Table 1. Histologic types of gastric carcinoma

\begin{tabular}{lc}
\hline Histologic type & $n(\%)$ \\
\hline Well differentiated & $931(21.8)$ \\
Moderately differentiated & $1017(23.8)$ \\
Poorly differentiated & $1459(34.2)$ \\
Signet-ring cell & $397(9.3)$ \\
Papillary & $339(7.9)$ \\
Mucinous & $112(2.6)$ \\
Special type & $17(0.4)$ \\
Total & $4272(100)$ \\
\hline
\end{tabular}

were seen between the MUC and NMUC groups in sex distribution or synchronous liver metastasis.

\section{Survival}

The overall survival rate (1 patient was lost to follow-up in the MUC group and 25 were lost to follow-up in the NMUC group) was significantly lower in MUC than in NMUC patients $(P<0.05)$; the 5-year survival rate was $64.7 \%$ in MUC patients and $75.6 \%$ in NMUC patients (Fig. 1). However, when the patients were stratified according to stage there was no significant difference in survival rate at each stage between the two groups (in 61 NMUC patients, stage was not known) (Fig. 2).

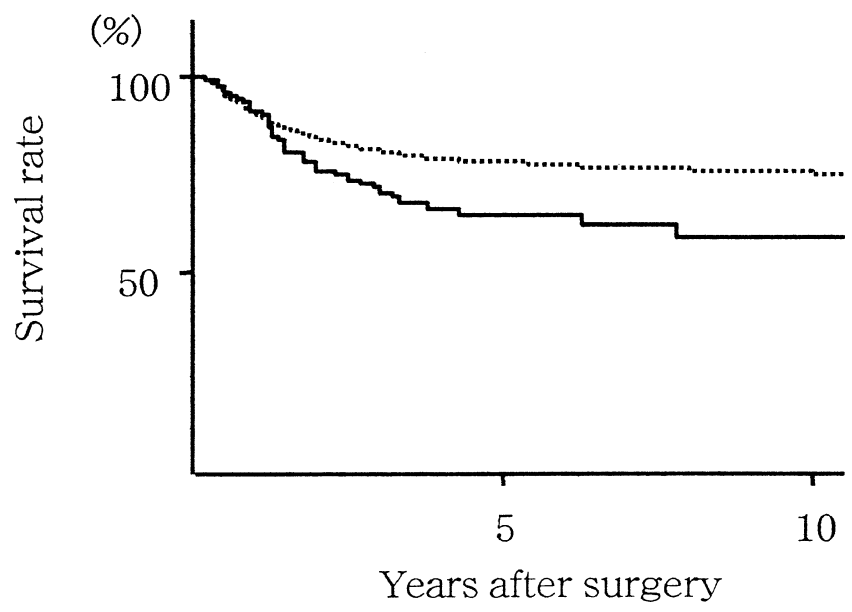

Fig. 1. Survival curves for patients with mucinous adenocarcinoma (MUC; solid line; $n=111)$ and nonmucinous adenocarcinoma (NMUC; dashed line; $n=4135) . P<0.05$

Table 2. Clinicopathologic findings of mucinous adenocarcinoma (MUC) and nonmucinous adenocarcinoma (NMUC)

\begin{tabular}{lccc}
\hline & MUC $(n=12)$ & NMUC $(n=4160)$ & $P$ \\
\hline Age & $56.0 \pm 12.0$ & $58.9 \pm 11.3$ & $<0.05$ \\
Sex & & & \\
$\quad$ Male & $73(65.2 \%)$ & $2791(67.1 \%)$ & NS \\
$\quad$ Female & $39(34.8 \%)$ & $1369(32.9 \%)$ & \\
Location & & & \\
$\quad$ Upper third & $8(7.1 \%)$ & $603(14.5 \%)$ & $<0.05$ \\
$\quad$ Middle third & $35(31.3 \%)$ & $1856(44.6 \%)$ & \\
$\quad$ Lower third & $69(61.6 \%)$ & $1701(40.9 \%)$ & \\
Tumor size (mm) & $60.1 \pm 31.3$ & $44.7 \pm 34.4$ & $<0.05$ \\
Depth of invasion & & & \\
$\quad$ To or beyond muscularis propria & $89(79.5 \%)$ & $1766(42.5 \%)$ & $<0.05$ \\
Lymph node metastasis & $71(63.4 \%)$ & $1467(35.3 \%)$ & $<0.05$ \\
Lymphatic permeation & $79(70.5 \%)$ & $1611(38.7 \%)$ & $<0.05$ \\
Venous permeation & $28(25.0 \%)$ & $694(16.7 \%)$ & $<0.05$ \\
Peritoneal dissemination & $22(19.6 \%)$ & $257(6.2 \%)$ & $<0.05$ \\
Synchronous liver metastasis & $1(0.9 \%)$ & $81(1.9 \%)$ & $\mathrm{NS}$ \\
Curative resection & $83(74.1 \%)$ & $3812(91.6 \%)$ & $<0.05$ \\
\hline
\end{tabular}

NS, Not significant 

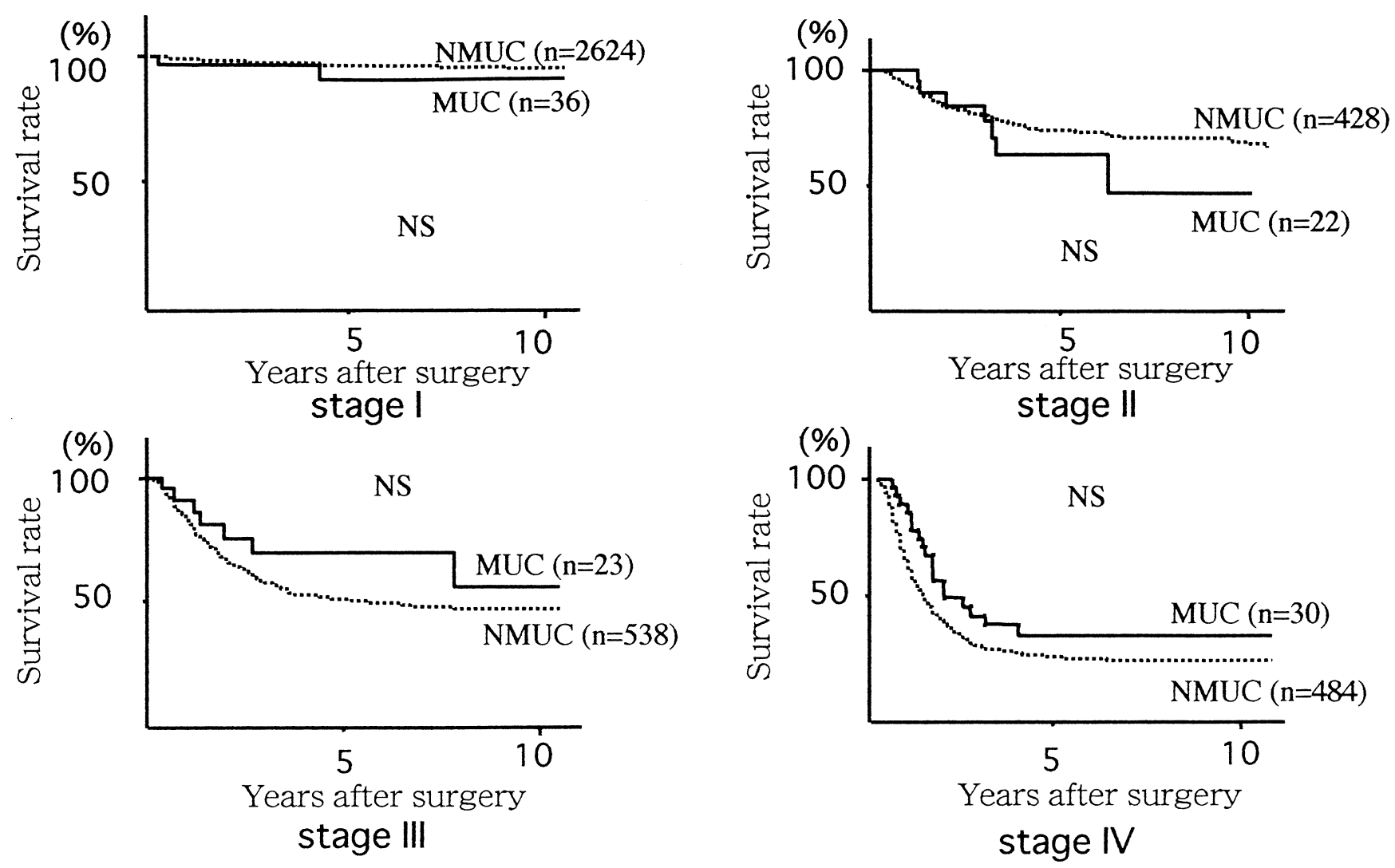

Fig. 2. Survival curves according to stage in patients with mucinous adenocarcinoma (MUC) and nonmucinous adenocarcinoma (NMUC). NS, not significant

\section{Cancer depth and lymph node metastasis}

Rates of lymph node metastasis in MUC patients were: $0 \%(0 / 8$ patients), in those with depth to the mucosa or muscularis mucosa $(\mathrm{m}) ; 26.7 \%(4 / 15)$ in those with depth to the submucosa $(\mathrm{sm}) ; 50 \%(8 / 16)$ in those with depth to the muscularis propria (mp); $75 \%(15 / 20)$ in those with depth to the subserosa (ss); 85.1\% (40/47) in those with depth to the serosa (se); and 66.7\% (4/6) in those with depth to adjacent structures (si). Rates of lymph node metastasis in NMUC patients were: $6.0 \%(75 / 1259)$ in those with depth to the $\mathrm{m} ; 8.9 \%$ (101/ $1135)$ in those with depth to the sm; $52.3 \%$ (194/371) in those with depth to the mp; $67.1 \%(318 / 474)$ in those with depth to the ss; $84.8 \%(684 / 807)$ in those with depth to the se; and $83.3 \%(95 / 114)$ in those with depth to si.

As for the relationship between cancer depth and lymph node metastasis, the frequency of metastasis increased as the cancer invaded to the submucosa in MUC patients, compared with NMUC patients $(P<$ 0.05 ), while, in NMUC patients, the frequency of lymph node metastasis increased as the cancer invaded to the muscularis propria compared with MUC patients (Fig. $3)$.

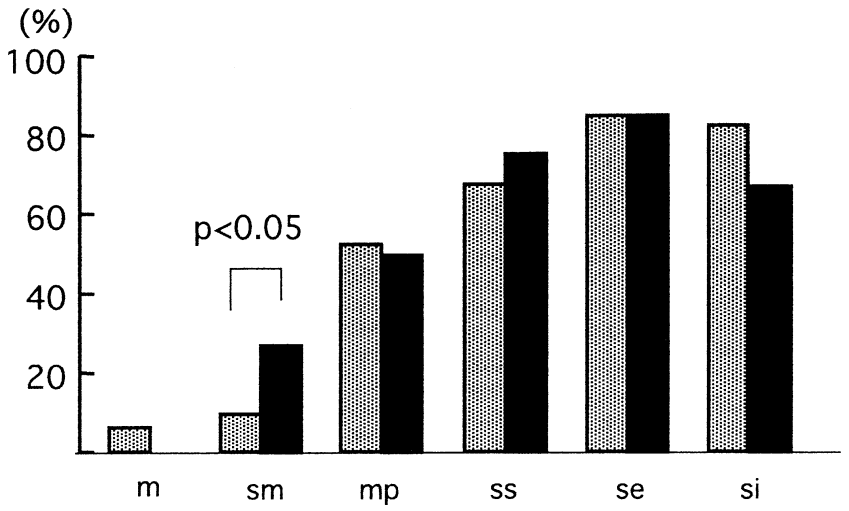

Fig. 3. Cancer depth and lymph node metastasis of mucinous adenocarcinoma (MUC; black bars) and nonmucinous adenocarcinoma (NMUC; stippled bars). $m$, Mucosa or muscularis mucosa; $s m$, submucosa; $m p$, muscularis propria; ss, subserosa; $s e$, penetration of serosa; $s i$, invasion of adjacent structures

\section{Discussion}

MUC is, reportedly a rare histologic type $(2.4 \%-4.9 \%)$ of gastric cancer [5-12], and its clinicopathologic features and prognosis have not yet been fully clarified. In 
this study, we investigated 112 MUC patients, perhaps the largest number of test patients reported in 15 years, and we presume that this resulted in some clarification of MUC characteristics.

Compared with NMUC, MUC clinicopathologic features are reported to include: larger size $[6,7,9,12]$; deeper invasion (advanced stage in $88.5 \%-94.8 \%$ of the patients) [5-7,9]; higher rate of lymph node metastasis $(80.3 \%-82.0 \%)$ [9,10,12]; and higher rate of peritoneal dissemination $(19.7 \%-21 \%)[9,10]$, features that correspond to the results of our present study. But other clinicopathologic factors are still controversial. The mean age of MUC patients has been reported as 51.663.2 years [5-12]. We and other investigators $[7,8]$ have reported that MUC is frequently observed in younger patients, however, some reports show no significant differences in MUC incidence by age [5,6,8,9,12]. Although most MUC is reported to be located in the lower third of the stomach [6,7], other reports show no difference in tumor location $[5,8,10]$. Very few reports show lymphatic and venous permeation of MUC. Adachi et al. [8] examined 42 patients with MUC and 73 control patients with NMUC. They reported that lymphatic permeation was observed more often in MUC than in NMUC patients $(83 \%$ vs $66 \%)$, but that the rate of venous permeation was not significantly different between the two groups (12\% vs $23 \%)$. However, the results of our present study showed that both lymphatic and venous permeation were more frequent in MUC than in NMUC patients. Kawamura et al. [9] investigated 61 MUC patients and 748 patients with NMUC and reported a lower rate of liver metastasis in MUC than in NMUC patients (1.6\% vs $4.5 \%)$. However, we found no significant differences in liver metastasis rates between MUC and NMUC patients.

In regard to prognosis, the reported 5-year survival rates of MUC patients in various studies range from 18.0 to $45.1 \%$. There are two opinions regarding their prognosis; one opinion indicates no prognostic differences between MUC and NMUC patients (e.g., as shown by Sassa and Kino [5] and Adachi et al. [8]). On the other hand, Takeshita et al. [7], Koufuji et al. [10], and $\mathrm{Wu}$ et al. [12] reported that MUC patients showed poorer prognosis than NMUC patients. The results of our present study indicated significantly poorer prognosis in MUC than in NMUC patients. This is, possibly, because no prognostic difference was seen in patients at each stage, suggesting that MUC patients are likely to have advanced cancer.

Sassa and Kino [5] reported a relationship between cancer depth and lymph node metastasis in MUC, showing that the frequency of lymph node metastasis according to cancer depth was $33.3 \%$ for sm; $55.6 \%$ for $\mathrm{mp} ; 66.7 \%$ for ss; and $73.6 \%$ for se. Yamagiwa [6] reported a frequency of lymph node metastasis of $13 \%$ for $\mathrm{sm} ; 36 \%$ for $\mathrm{mp}$; and $70 \%$ for ss or beyond. We compared rates of lymph node metastasis in MUC and NMUC patients according to cancer depth. The results showed that, in MUC patients, lymph node metastasis was more frequently seen in patients with a cancer depth of sm or more.

In conclusion, although many of MUC are still unclear, we found that this carcinoma, compared with NMUC, appeared to have the following aspects characteristics: (1) more frequent occurrence in younger patients; tumor location at a lower site in the stomach; larger tumor diameter; deeper cancer depth; higher incidence of lymph node metastasis, lymphatic and venous permeation, and peritoneal dissemination; and poorer prognosis. (2) The prognosis of MUC is poor because most of the MUC is advanced. (3) There is a possibility of lymph node metastasis in patients with a cancer depth of sm or more, and great attention should be paid to this.

\section{References}

1. Secco GB, Fardelli R, Campolra E, Lapertosa G, Gentile R, Zoli $\mathrm{S}$, et al. Primary mucinous adenocarcinoma and signet-ring cell carcinomas of colon and rectum. Oncology 1994;51:30-4.

2. Hara Y, Ogata Y, Okita A, Inuzuka K, Konishi J, Kanazawa M, et al. A clinicopathological study of mucinous carcinoma in the colon. J Jpn Surg Assoc 1998;59:2981-5.

3. Ikegawa R, Nakae S, Nakamura T, Tabuti Y. Clinicopathological study on mucinous carcinoma of colon and rectum. J Jpn Soc Colo Proctol 1999;52:98-106.

4. Consorti F, Lorenzotti A, Midiri G, Di Paola M. Prognostic significance of mucinous carcinoma of colon and rectum: a prospective, case-control study. J Surg Oncol 2000;73:70-4.

5. Sassa H, Kino I. A comparative study on mucinous carcinoma of the stomach and large intestine. Jpn J Gastroenterol 1979;76:6597.

6. Yamagiwa H. Colloid carcinoma of gastrointestinal tract. 1. Stomach. Jpn J Clin Pathol 1985;8:924-8.

7. Takeshita K, Kando F, Maruyama M, Ochi K, Sunagawa M, Habu $\mathrm{H}$, et al. Clinocopathlogical evaluation on mucinous adenocarcinoma of the stomach. Jpn J Gastroenterol Surg 1986;19:171117.

8. Adachi Y, Mori M, Kido A, Shimono R, Maehara Y, Sugimachi K. A clinocopathologic study of mucinous gastric carcinoma. Cancer 1992;69:866-71.

9. Kawamura M, Sato T, Tsusima H, Yokokawa T, Marumori K, Iseki M. Clinicopathological study of mucinous adenocarcinoma of the stomach. Jpn J Gastroenterol Surg 1994;27:10-16.

10. Koufuji K, Takeda J, Toyonaga A, Kodama I, Aoyagi K, Yano S, et al. Mucinous adenocarcinoma of the stomach. Clinocopathological studies. Kurume Med J 1996;43:289-94.

11. Songür Y, Okai T, Watanabe H, Fujii T, Motoo Y, Sawabu M. Preoperative diagnosis of mucinous gastric adenocarcinoma by endoscopic ultrasonography. Am J Gastroenterol 1996;91:158690.

12. Wu C-Y, Yeh H-Z, Shih R T-P, Chen G-H. A clinicopathologic study of mucinous gastric carcinoma including multivariate analysis. Cancer 1998;83:1312-8.

13. Japanese Research Society for Gastric Cancer. Japanese classification of gastric carcinoma. 2nd English ed. Gastric Cancer 1998;1:10-24. 